DOCTRINA

\title{
La COP25 en Madrid: Deudas, dudas y el futuro de la acción climática oceánica mundial
}

\author{
COP25 Madrid: Debts, doubts and the future of global oceanic climate action
}

\author{
Pablo Carrasco Hernández \\ Investigador independiente
}

RESUMEN El propósito de este trabajo es analizar los avances y desafíos de la $\mathrm{COP}_{25}$ en Madrid. Si bien la conferencia estuvo marcada por las negociaciones en torno al polémico artículo 6 del Acuerdo de París, sus críticos argumentan que los esfuerzos esbozados contra el cambio climático no lograron dar la urgencia necesaria a las políticas oceánicas y glaciares. Sobre esa base, este artículo no solo revisará los esfuerzos internacionales en esta materia desde la COP21 de París hasta la COP24 de Katowice, sino los esfuerzos por dar seguridad al océano y a la Antártida en Chile. Todo esto se hará considerando la Política Oceánica Nacional, aprobada en 2018, y el papel de la Ley Marco de Cambio Climático, que el actual gobierno ha impulsado a través de la articulación entre el sector público, privado, académico y otros actores competentes para una mejor gestión oceánica en Chile. Finalmente, se advierte que el punto más deficiente de este hito fue no haber considerado a los océanos como parte integral del problema de los efectos de los gases invernadero. Esto determinó que la $\mathrm{COP}_{25}$ fuera catalogada como un fracaso.

PALABRAS CLAVE COP25, artículo 6, Acuerdo de París, océanos, glaciares, Ley Marco de Cambio Climático.

ABSTRACT The main purpose of this work is to analyze the progress and challenges of the previous $\mathrm{COP}_{25}$ version finally developed in Madrid, which although it was marked by the negotiations around the controversial Article 6 of the Paris Agreement, its critics argue that their efforts to in the fight against climate change, they failed to give urgency to oceanic and glacial policies. On this basis, this article will review international efforts in this area from $\mathrm{COP}_{21}$ in Paris to $\mathrm{COP}_{24}$ in Katowice, as well as efforts to provide security to the ocean and Antarctica in our country considering the National Oceanic Policy approved in 2018, and the role of the Framework Law on Climate Change that the current government has promoted through the articulation between the public, private, academic and competent actors for better ocean management in Chile. Finally, it is noted that the lowest point was not having considered the oceans in an integral way 
to the problem of the effects of GHGs, which has determined that the last $\mathrm{COP}_{25}$ in Madrid is classified as a failure.

KEYWORDS COP25, article 6, Paris Agreement, oceans, glaciers.

\section{Introducción}

El capitalismo y el altruismo son mutuamente excluyentes. -Ayn Rand, filosofa ruso-norteamericana.

Con respecto al futuro del medio ambiente en Chile, el $35 \%$ de los encuestados se declara ni muy optimista ni muy pesimista, mientras que el 10\% de los consultados se siente o muy optimista o muy pesimista. ${ }^{1}$

En el contexto del estallido social que aún remece tanto la estabilidad y legitimidad política e institucional del gobierno de Chile como su modelo de desarrollo, el presidente Sebastián Piñera decidió no celebrar la COP25 en Santiago de Chile. A partir de esto, por urgencia de las autoridades nacionales y de la Secretaría General de las Naciones Unidas, se decidió que la conferencia se trasladara a Madrid. En este contexto, y considerando la urgencia ambiental a nivel mundial, la COP25 era una oportunidad única para crear los diálogos y puentes de acuerdo necesarios entre las naciones ante la amenaza del cambio climático. El objetivo era crear un mecanismo que fiscalizara los mercados de carbono y, de manera más urgente, que se redujeran las emisiones globales de dióxido de carbono a la atmósfera antes de 2030. Estos objetivos fueron advertidos por los especialistas de la Organización de las Naciones Unidas y los miembros de la comunidad científica internacional de forma clara antes, durante y después de la conferencia.

En ese momento, y luego de la intervención pública de la activista ambiental sueca de 17 años Greta Thunberg, la sociedad civil no solo insistía en la inacción de las naciones desarrolladas para destrabar las negociaciones en torno a la reducción de las emisiones de gases de efecto invernadero, sino también en recalcar que el actual modelo económico de desarrollo privilegiaba a las industrias extractivas - conocidas como comodities - en naciones en vías de desarrollo, por sobre un modelo de crecimiento sostenible que garantizara el resguardo de los elementos naturales. Por otra parte, las reflexiones también giraban en torno al modelo de consumo capitalista, que ha llevado hasta el límite la producción económica en industrias clave como la

1. Dirección de Estudios Sociales, Instituto de Sociología, Informe Final «Encuesta Nacional de Medio Ambiente 2018», 2018, p. 43, disponible en bit.ly/2VxlIPw. 
minería, la agroindustria y el sector energético de hidrocarburos. En relación con este último aspecto, es importante mencionar que países como China, India y Estados Unidos sostienen su crecimiento en el producto interno bruto, en base al desarrollo de estos sectores productivos.

$\mathrm{Al}$ asumir Chile la presidencia de la conferencia, con la ministra de Medio Ambiente Carolina Schmidt a la cabeza de las negociaciones, el objetivo fue conciliar los intereses de los países europeos y en vías de desarrollo con las advertencias y los datos que entregaba la comunidad científica por sobre los intereses de las naciones industrializadas y sus compañías responsables, en gran medida, de las altas emisiones de dióxido de carbono a la atmósfera. De esta forma, esta instancia debía capitalizar las ambiciones y esperanzas que la comunidad civil internacional y científica habían depositado en ella. La idea era tratar el tema de la negociación del artículo 6, fundamental para fiscalizar, controlar y reformar el sistema de mercados de bonos de carbono, y dotarlo de una institucionalidad eficiente, eficaz y transparente a futuro en torno a un Acuerdo de París que, incluso actualmente, no encuentra respuestas en los gobiernos nacionales.

Tras una jornada de maratónicas negociaciones, y después de la exclusión de un primer borrador calificado de «insuficiente», se llegó a un acuerdo denominado «Chile-Madrid. Tiempo de Actuar». Si bien en él se pedía aumentar la «ambición climática» en 2020 y cumplir con el Acuerdo de París para evitar que el aumento de la temperatura superara los $1,5^{\circ} \mathrm{C}$ este siglo, sus críticos aseguraron que fue un consenso «débil», ya que, entre otras cosas, no se incluía el apartado sobre la regulación de los mercados de carbono porque los países no lograron llegar a un compromiso común. En consecuencia, el resultado fue un documento propositivamente débil, ya que evitó abordar los puntos clave para la COP26 en Glasgow, como el mercado de carbono y la discusión en torno a los océanos y glaciares, dos aspectos de vital importancia tanto para los activistas como para la comunidad científica.

Frente a la pregunta de si esto puede considerarse como un fracaso o incluso una catástrofe para la protección del clima, entre los posibles resultados previsibles de la conferencia, el comercio de los derechos de emisión fue algo positivo, ya que los Estados decidieron intensificar de inmediato y considerablemente sus esfuerzos para la protección del clima a través del apoyo solidario, financiero y a largo plazo tanto a los países ribereños como a las naciones en vías de desarrollo, propiciando mejores medidas de adaptación al cambio climático.

No obstante, no hay que perder de vista que el acuerdo buscaba esclarecer y fijar las «reglas del juego» del comercio internacional de emisiones del artículo 6 del Acuerdo de París y que, gracias a la resistencia de una coalición de Estados especialmente vulnerables y de la Unión Europea, el intento por acordar normas, que habrían hecho de este comercio de emisiones un enorme fraude para la opinión pública internacional, fue rechazado en Madrid. De hecho, Laurence Tubiana de la Fundación Europea del 
Clima y arquitecto del Acuerdo de París, afirmó lo siguiente: «los principales actores no cumplieron con las expectativas, pero gracias a una alianza progresiva de pequeños Estados insulares, países europeos, africanos y latinoamericanos, obtuvimos el mejor resultado posible contra la voluntad de los grandes contaminadores». ${ }^{2}$

El problema de los océanos y su vinculación con la crisis climática mundial fue un tema transversal en los side events de la $\mathrm{COP}_{25}$, ya que la población mundial depende de los océanos de más maneras de las que se piensa: no solo proporciona materias primas y alimentos, sino que es clave como fuente del oxígeno que necesitamos para vivir (una de cada dos inspiraciones viene de los océanos) y modera las temperaturas globales. Los océanos, las zonas costeras y sus ecosistemas no solo son un inmenso reservorio de biodiversidad, también proporcionan valiosos servicios a los seres humanos. A modo de ejemplo, el sector de la pesca y de la acuicultura sustentan los medios de vida de entre el $10 \%$ y $12 \%$ de la población mundial, lo que hace urgente una política de acción climática que preserve los hábitats marinos y las especies que albergan.

Ahora bien, Chile se ha identificado como un país comprometido con la reducción de sus propias emisiones, con cooperar con sus propias contribuciones (INDC) a nivel continental y como uno de los promotores en la COP21 en París. Sin embargo, y en el contexto de la preparación de la $\mathrm{COP}_{25}$, el país no podría ser considerado como un «líder internacional» en la lucha contra el cambio climático, ya que aún existen asuntos nacionales pendientes y «en el gobierno asumen que resolverlos, o no, puede marcar el éxito o el fracaso de la cumbre en nuestro país», ${ }^{3}$ ya que la escasa experiencia gubernamental en esta materia - desde 1990 con la vuelta a la democracia - ha pesado en su displicente manejo en temas en los que la sociedad civil y los especialistas llaman a actuar de inmediato, considerando, por ejemplo, la negativa del actual gobierno a firmar y ratificar el Acuerdo de Escazú, mecanismo que facilitaría la resolución de conflictos ambientales a nivel nacional y territorial, lo que, según la Comisión Interamericana de Derechos Humanos, sería de suma urgencia en un país con más de cien conflictos de esta naturaleza.

La relevancia de los océanos en esta discusión tiene que ver con las conclusiones de los especialistas que componen el Grupo Intergubernamental de Expertos sobre el Cambio Climático, ya que fueron alarmantes porque la absorción de dióxido de carbono por parte de los océanos está causando, además, la acidificación de sus aguas, lo que pone en riesgo la vida marina. Esto no solo es extremadamente nocivo desde el

2. «COP25: 3 claves del polémico acuerdo por el clima (y porqué dicen que fracasó)», $B B C, 15$ de diciembre de 2019, disponible en bbc.in/2AdiGZp.

3. Jorge Molina, «Patricia Espinosa fija meta para la COP25: «Es absolutamente indispensable que logremos un resultado en Santiago en relación con el artículo 6»", País Circular, 27 de junio 2019, disponible en bit.ly/3g950wk. 
punto de vista ecológico, sino que trae aparejado riesgos a la seguridad alimentaria, ya que se proyecta una disminución de captura de las pesquerías. Sobre esto, la ciencia recabó más datos y sus especialistas fueron tomados en cuenta. Además, se logró un cambio en el paradigma que consideraba que el aire y la emisión de gases de efecto invernadero eran lo único grave que las acciones humanas generan en el ambiente.

Esta era la «COP Azul» del gobierno, en la se esperaba cimentar las bases de una política oceánica hacia el futuro, coincidentes con los anhelos de la sociedad civil y tanto con las advertencias de especialistas como de científicos para evitar una mayor degradación de los océanos y de las masas glaciares del Polo Norte y Sur. Sin embargo, debido a la mala gestión de la presidencia de la $\mathrm{COP}_{25}$ y la falta de acuerdo entre medidas de adaptación, mitigación y financiamiento de la acción climática, ni los negociadores ni el equipo chileno pudieron revertir un desastre que, a todas luces, era esperable debido a la falta de preparación para esta crucial instancia negociadora.

No basta que el océano sea considerado como un área que proporciona elementos naturales para ser consumidos por la población humana. En lugares como Paipote en la región de Antofagasta, ante la escasez de agua potable en el interior de la región, la planta desaladora del pequeño pueblo de pescadores proporciona aproximadamente un $78 \%$ a un $80 \%$ del agua potable requerida, siendo una de las 24 que actualmente funciona en todo el país, ${ }^{4}$ lo que implica la producción de 5.570 litros por segundo, cifra que se incrementará en un $160 \%$ en los próximos cinco años. Todo esto ocurre mientras se espera la conclusión de otros 22 proyectos, lo que constituye una verdadera oportunidad para mitigar los efectos de la megasequía en el norte de Chile.

La COP25, enfocada en los océanos bajo el nombre de «COP Azul», trabajó cinco ejes claves:

- Un «eje saludable» para mitigar los efectos de la contaminación de los mares y la preservación de especies marinas.

- Ser un plan sustentable para las actividades económicas del mar como la industria acuícola y pesquera, proyectos de energía sustentable y turismo.

- Desarrollar el concepto de «océano seguro», considerando el resguardo de la navegación, la protección del borde costero y la gestión institucional ante desastres naturales.

- Instrucción formativa en temas oceánicos a trabajadores del sector industrial y artesanal de pesca y acuicultura.

- Desarrollo de una «gobernanza oceánica» que fomente la creación de un nuevo marco normativo y fórmulas de participación ciudadana.

4. Todas concentradas en la región de Arica y Parinacota, la región de Tarapacá y la región de Coquimbo. 
No obstante, los intereses de los países fundamentalmente ribereños y sus demandas por incluir temas en sus agencias nacionales chocaron tanto con el Acuerdo de París como con las aristas clave que permiten la ratificación de los cambios y negociaciones. Sobre esto, existe una visión alternativa que señala que, en un hito preCOP en abril de 2019 en Madrid, especialistas y representantes de organizaciones no gubernamentales y de la sociedad civil advirtieron que esta reunión era verdaderamente una disputa para ganar fuerza política y credibilidad.

\section{Desde el Acuerdo de París hasta la COP24 de Katowice}

La Cumbre de París de 2015 se caracterizó por tres grandes virtudes. La primera, por haber reunido a 159 naciones para definir, bajo un consenso político, un plan estratégico de cinco años para tratar de luchar contra las emisiones de gases de efecto invernadero y sus efectos en todo el planeta a través de la incorporación de medidas de mitigación, adaptación y resilencia. La segunda, por las medidas de mitigación o reducción del dióxido de carbono del océano y del uso que hacemos de él, específicamente contra la acidificación de los mares producto de la acción industrial y de los efectos de los gases de efecto invernadero; por el impulso a las energías renovables basadas en el viento, olas o mareas para evitar la carbono dependencia en sectores estratégicos de países subdesarrollados como China o potencias industriales como Estados Unidos; y por incentivar un nuevo modelo de desarrollo y de eficiencia energética que estimule la inversión en energías limpias y a bajo costo. La tercera, porque el plan estratégico deberá incluir la creación de capacidades de investigación oceánica para los países que no dispongan de ella, en base al financiamiento desde las naciones desarrolladas y la exportación e intercambio de conocimiento para abordar la mejora de la capacidad de observación de los impactos del cambio climático en los océanos, así como contemplar los aspectos de educación, participación social y ciencia ciudadana.

No obstante, más allá de este plan estratégico de la Cumbre, sobresale el Acuerdo de París, que ha caracterizado a la acción climática en los últimos años. Su objetivo principal es evitar que el incremento de la temperatura media global supere los $1,5^{\circ} \mathrm{C}$ o $2^{\circ} \mathrm{C}$ con respecto a los niveles preindustriales. Además, busca promover esfuerzos adicionales que hagan posible que el calentamiento global no supere $\operatorname{los} 1,5^{\circ} \mathrm{C}$, lo que impediría que las emisiones globales toquen techo a partir de la transición hacia una matriz energética «limpia» antes de 2030, junto con una política pública ambiental eficiente, eficaz y transparente.

En el Informe especial sobre el océano y la criósfera en un clima cambiante del Grupo Intergubernamental de Expertos sobre el Cambio Climático de 2019, se expone que el océano se ha llevado la peor parte de la crisis, provocando la acidificación, el sobrecalentamiento y la desoxigenación de los mares a un ritmo frenético, impre- 
decible y peligroso. Estos son los tres factores presentes en todo evento de extinción masiva en la historia de la Tierra, que ponen en peligro a arrecifes de coral y especies de crustáceos como cangrejos de agua dulce y mariscos bivalvos.

Sin embargo, la acción climática sobre las emisiones de gases de efecto invernadero solo se constituyó como uno de los puntos principales para alcanzar mínimos acuerdos para la acción climática (desde la $\mathrm{COP}_{21}$ hasta Katowice), y no han sido suficientes para cambiar el rumbo de la dudosa acción climática mundial, ni tampoco para alterar las bases del modelo de desarrollo capitalista extractivista no solo de Chile, sino de las potencias industriales como China, Estados Unidos o India.

La apuesta de la presidencia de la COP21 en París, en ese entonces encabezada por Laurent Fabius, ex primer ministro de Francia durante el gobierno de François Mitterrand, era precisa y contundente. Consistió en aunar los esfuerzos de los países a través de negociaciones que concluyeran en acuerdos vinculantes para contrarrestar las emisiones globales antes de que en 2020 llegasen a un tope máximo tolerable para las ambiciones de los países más vulnerables, como las naciones ribereñas e isleñas, y las naciones en vías de desarrollo, como Chile (siendo de las naciones más expuestas a las consecuencias de no controlar las emisiones globales de dióxido de carbono de las naciones industrializadas); y considerar a los océanos como el gran factor que pone en peligro la acción climática mundial.

El acuerdo ha establecido un sistema de revisión cada cinco años que no solo permita determinar la evolución de los INDC’s (Intended Nationally Determined Contribution) de cada país, sino incrementar su ambición para asegurar que se alcance el objetivo de mantener el aumento de la temperatura por debajo de los $2^{\circ} \mathrm{C}$. Sin embargo, el primer balance no se produciría hasta 2023 (con una valoración inicial en 2018), y la primera revisión oficial se ha retrasado hasta 2025. Estas fechas, según muchos especialistas, son tardías. De hecho, en opinión de Laurent Fabius: «el agua está en la parte medular de todo y se debe actuar en dos planos: limitar el calentamiento climático, lo cual tendrá un efecto directo sobre los océanos, y aumentar los esfuerzos de adaptación a los efectos del cambio» sobre los océanos. ${ }^{5}$

$\mathrm{Al}$ respecto, se ha indicado que los INDC's:

De cada parte representarán una progresión con respecto a la contribución determinada a nivel nacional que esté vigente para esa parte y reflejará la mayor ambición posible de dicha parte, teniendo en cuenta sus responsabilidades comunes pero diferenciadas y sus capacidades respectivas, a la luz de las diferentes circunstancias nacionales. ${ }^{6}$

5. Romain Clergeat y François de Labarre, «Laurent Fabius, presidente de la COP21, habla de los retos vitales relacionados con el agua en una entrevista concedida a Paris Match para su número especial «agua», Ministère de l'Europe et des Affaires étrangères, 4 de octubre de 2017, disponible en bit.ly/2Vo2xri.

6. Para más información, véase el artículo 4.3 del Acuerdo de París, disponible en bit.ly/2VAfT $3 \mathrm{X}$. 
Todo esto, considerando los desafíos a nivel regional, nacional y global ante las medidas de mitigación y adaptación. Meses antes de la COP25, la exministra Schmidt mencionó que el desafío era aumentar la ambición, terminar la negociación y entrar derechamente a la etapa de implementación de lo acordado, ${ }^{7}$ pero la institucionalidad del país y los negociadores chilenos tendrían la misión de liderar las negociaciones de los temas de interés de la agenda internacional, no solo de los del gobierno.

$\mathrm{Si}$ bien a partir del Acuerdo de París Chile instaló en la rondas de negociaciones tanto los temas de medidas de adaptación como de protección de los océanos a través de la plataforma G2o y de que, en palabras del actual presidente Sebastián Piñera, el país no solo tenía un compromiso para reducir sus emisiones entre un 30\% y un $45 \%$ antes de 2030, sino también para impulsar con fuerza y decisión la transición energética y el impulso de las energías renovables no convencionales, ${ }^{8}$ lo cierto es que, según el III Informe Bienal (2018):

Se requiere que las políticas de todos los sectores emisores estén alineadas con la mitigación. En consecuencia, propone fortalecer y formalizar la base institucional de la política sobre cambio climático, adoptar e implementar un conjunto de políticas nacionales sobre cambio climático, y ejecutar un marco de monitoreo y evaluación de las políticas de adaptación y mitigación. ${ }^{9}$

Además, el Acuerdo de París es coherente con una política que establece la necesidad de una transición hacia modelos de energías renovables de carbono neutralidad a largo plazo, ya que para ello se cuenta con un importante paquete financiero que pretende ayudar a su implementación, y deberá construirse sobre la base del objetivo, para los países desarrollados, de movilización de 100 mil millones de dólares anuales a partir de 2020 (denominada ambición preCOP 2020). Todo esto a través de distintas fuentes, como transferencias de tecnologías y de fondos de inversiones basadas en la cooperación.

En la cita de la COP22 de Marrakech, se depositaron muchas expectativas por comenzar a trabajar de forma activa sobre las bases del Acuerdo de París. En un primer momento, en la convención se pretendía fijar acciones concretas para reducir la emisión de dióxido de carbono a la atmósfera y, de este modo, empezar a frenar el cambio climático. Sin embargo, de nuevo se trabajó más sobre la base teórica y política, lo que ha dio como resultado el «Decreto de Marrakech», un documento que explica a las

7. Giovanni Calderón, «COP25: Negociaciones contra reloj», País Circular, 22 de julio 2019, disponible en bit.ly/2CQFDT7.

8. «Chile en el G2o: Presidente reafirma el compromiso del país con el Acuerdo de París», Diario UChile, 1 de diciembre 2018, disponible en bit.ly/2VtZsGb.

9. Ministerio de Medio Ambiente de Chile, «III Informe Bienal de Actualización de Chile sobre Cambio Climático», 2018, p. 117, disponible en bit.ly/2VAoP6a. 
partes cómo elaborar el plan de actuación y las estrategias para conseguir los objetivos pactados en el contexto de la Convención en París. A pesar de esto, es importante mencionar que este esfuerzo fue infructuoso debido a la decisión de países como Brasil de detener las negociaciones y dejar el acuerdo sin demasiadas alteraciones.

A pesar de que esta cumbre fue una reunión de dos semanas para definir las reglas del juego de la aplicación del Acuerdo de París, se lograron algunas decisiones concretas. Una de ellas fue la alianza impulsada por el Reino Unido y Canadá para deshacerse gradualmente del carbón antes de 2030. Más de 20 países y Estados firmaron este documento. Sin embargo, Estados Unidos, China, India, Alemania o Australia, algunos de los principales consumidores de carbón, no están presentes en la alianza, pero sí lo están países como Costa Rica, México y los estados norteamericanos de Oregón y Washington.

En la Cop24 en Katowice, los representantes de las 197 naciones reunidas en Polonia lograron sellar las reglas que harían posible la implementación del Acuerdo de París. Lo pactado por la comunidad internacional fue consignado en un documento denominado como el «Libro de Reglas», el cual marcaría las pautas en los procesos para controlar el calentamiento global en los próximos años.

En el marco de las necesidades por establecer las medidas de mitigación, adaptación y resiliencia de Chile con respecto a las exigencias ratificadas por el país a partir del Acuerdo de París, si bien las emisiones nacionales son aproximadamente de $0,25 \%$ al 2015, se comprometió lo siguiente:

- Una meta de intensidad de carbono expresada en emisiones de gases de efecto invernadero por unidad de producto interno bruto nacional, como lo establece el compromiso chileno de NDC.

- Una meta expresada en toneladas de dióxido de carbono equivalente del sector del cambio de uso de la tierra y la silvicultura. ${ }^{10}$

A partir del Acuerdo de París, todas la partes, y en especial Chile, han aceptado aumentar la capacidad de adaptación, para lo cual establecen el objetivo mundial relativo ella. En el caso chileno, esto se materializó en el Plan Nacional de Adaptación al Cambio Climático. No obstante, la Organización para la Cooperación y el Desarrollo Económico establece ciertas recomendaciones en su Informe de evaluación de 2012, siendo la «educación, la capacitación y la concientización, medidas de información y capacitación en temas del cambio climático y en la elaboración de medidas apropiadas de adaptación», ${ }^{11}$ lo que en mayor medida permitiría fomentar la adaptación a los

10. Ministerio de Medio Ambiente de Chile, «III Informe Bienal de Actualización de Chile sobre Cambio Climático», 2018, p. 120, disponible en bit.ly/2VAoP6a.

11. Ministerio del Medio Ambiente, «Plan Nacional de Adaptación al Cambio Climático», 2016, p. 13, disponible en bit.ly/2ZszT9A. 
efectos del forzamiento radiativo directo, ya que entre 2011 y 2030 se proyectaba que los aumentos de temperatura fluctuaran entre los $0,5^{\circ} \mathrm{C}$ para la zona sur y los $1,5^{\circ} \mathrm{C}$ para la zona del norte grande y altiplánica. Por otra parte, para el período entre 2031 y 2050, se mantiene el patrón de calentamiento, pero con valores mayores». ${ }^{12}$

Según el V informe del Grupo Intergubernamental sobre el Cambio Climático de 2013, existen dos procesos antropogénicos que afectan el sistema climático, que se expresan por las diferencias entre la luz solar absorbida por la tierra y la energía desprendida de la tierra hacia el espacio. Estas influyen en la capacidad del sistema climático para equilibrar las emisiones de calor y dióxido de carbono de parte de las actividades económicas humanas. Estos procesos son:

- Forzamiento radiativo positivo o directo, que altera la temperatura de la superficie, ya que «es muy probable que las olas de calor se produzcan con más frecuencia y que sean de más duración, y que los eventos extremos de precipitación serán más intensos y frecuentes en muchas regiones» (Rodríguez, Picatoste y Heras, 2014: 35).

- Forzamiento radiativo negativo en el que, por el contrario, hay un enfriamiento de la tierra, haciendo "probable que las emisiones de óxidos de nitrógeno hayan inducido un forzamiento radiativo negativo neto». ${ }^{13}$

Todo esto depende de que los planes nacionales de adaptación contemplen un compromiso con un claro énfasis en la implementación y en la necesidad de planificar a mediano y largo plazo. En primer lugar, para lograrlo se requiere que cada parte no solo elabore, sino que también actualice regularmente planes y estrategias de adaptación y reporte sobre los avances en su implementación (artículo 7.9). Además, los esfuerzos de adaptación que realicen las partes deben ser informados mediante una comunicación sobre la adaptación e inscritos en un registro público (artículos 7.10 y 7.12). Para ello, como indica uno de los puntos del informe de evaluación de desempeño ambiental de Chile de 2016, siendo un carácter persistente del mismo, se necesita una mejor operatividad de las instituciones para fiscalizar, reportar y construir datos e información precisa al servicio de los planes nacionales de adaptación.

En segundo lugar, los mecanismos de flexibilidad del artículo 13, con el objetivo de aplicar políticas más resilientes en los países más vulnerables con respecto al cambio climático, establecen «un marco de transparencia reforzado para las medidas y el apoyo, dotado de flexibilidad para tener en cuenta las diferentes capacidades de

12. Ministerio del Medio Ambiente, «Plan Nacional de Adaptación al Cambio Climático», 2016, p. 16, disponible en bit.ly/2ZszT9A.

13. Grupo Intergubernamental de Expertos sobre el Cambio Climático, Summary for Policymakers, 2013, p. 13, disponible en bit.ly/2Zn>Wjz. 
las partes y basado en la experiencia colectiva».14 Además, estos mecanismos están basados en compromisos no formales sobre transparencia y confianza mutua. Sin embargo, aún no existen certezas de quiénes son los emisores y de si estos apoyos financieros no determinarán las acciones futuras del país en medidas de adaptación, en el caso de que se hayan realmente logrado estos compromisos en una fecha límite, lo que puede afectar la credibilidad de un país de cara a nuevas exigencias ambientales considerndo que los países receptores de los fondos deben rendir cuenta a la Secretaría de la organización de la COP y ante las demás partes del Acuerdo.

En relación con la COP21 en París, es importante mencionar que el resguardo de los océanos y su relación con las medidas de adaptación y mitigación ante las emisiones de dióxido de carbono de las potencias industriales como China, Estados Unidos e India nunca fue un tema de interés para las grandes naciones industrializadas. De hecho, la absorción de las emisiones por los océanos nunca generó interés y, por lo mismo, es relevante considerar que los problemas actuales de acidificación de los océanos se debe a que los mares absorbieron el 60\% del dióxido de carbono emitido antropogénicamente desde la Revolución Industrial (Di Pietro, 2019: 60). Si los océanos hubiesen estado lo suficiente saludables en términos de balance del ph del agua salada del mar, hubieran reducido entre el $80 \%$ o $90 \%$ del carbono y otros gases liberados en la atmósfera.

Además, desde la creación del Grupo Intergubernamental de Expertos sobre el Cambio Climático, y como efecto concreto de la masiva producción industrial de las naciones industrializadas - seguidas en menor medida en comparación con las naciones en vías de desarrollo que dependen de las actividades extractivas y de la dependencia del carbón para la generación de energía - resultado del cambio climático, «el nivel medio del mar se ha elevado 19 centímetros desde 1901, la cobertura de hielo en el ártico y Groenlandia ha perdido masa en todas las estaciones del año de manera sucesiva y los glaciares del hemisferio norte han disminuido». ${ }^{15}$

A pesar de esto, las expectativas, esperanzas y ansias por cambiar el rumbo de la acción climática mundial no solo se deshicieron como los glaciares ante el calor en la $\mathrm{COP}_{21}$ de París, sino también en Katowice, Polonia, denominada la capital europea del carbón. Es importante mencionar que la $\mathrm{COP}_{24}$ de Katowice se perfilaba como una instancia clave ante la urgencia mundial de tomar medidas para combatir el cambio climático y para «hacer funcionar» el Acuerdo de París, pero sus resultados decepcionaron. El informe del Grupo Intergubernamental de Expertos sobre el Cambio Climático da cuenta de que si se continúan emitiendo gases de efecto invernadero a la velocidad que se está haciendo, se llegaría al incremento de $1,5^{\circ} \mathrm{C}$

14. Para más información, véase el artículo 13.1 del Acuerdo de París.

15. Grupo Intergubernamental de Expertos sobre el Cambio Climático, Summary for Policymakers, 2013, p. 13, disponible en bit.ly/2Zn>Wjz. 
entre 2032 y 2050. Los riesgos asociados dependen de la magnitud del calentamiento según la ubicación geográfica, la vulnerabilidad, la implementación de medidas de adaptación y mitigación, y del nivel de desarrollo del país. Por eso, naciones latinas y pequeños países isleños o ribereños de Asia reúnen todas las condiciones para sufrir, año a año, los embates del clima, tan perfectamente relacionados con los embates del cambio climático.

Antes de la denominada «gran oportunidad» que tuvieron las naciones para concretar la acción climática como fue denominada la «COP Azul», la $\mathrm{COP}_{24}$ de Katowice en Polonia tampoco fue un halo de esperanza para los que pensaban que se acercaba el momento de cambiar el rumbo de las cosas. En este contexto, el Grupo Intergubernamental de Expertos sobre el Cambio Climático concluiría, el mismo año año de la celebración en Katowice, que sí se podía alcanzar el objetivo de impedir el aumento de los $1,5^{\circ} \mathrm{C}$, pero solo gracias a un fuerte compromiso, ya que (aún se espera que) antes de 2030 hacía falta reducir las emisiones en un $45 \%$ con respecto a los valores de 2010, además de llegar al balance de cero emisiones antes de 2050.

El objetivo era llevar a la práctica el Acuerdo de París, sobre todo considerando los amarres más cuestionados en torno a las negociaciones de los artículos 6.2 y 6.4 en cuanto a mecanismos de adaptación, mitigación y financiamiento (del que dependían las grandes instituciones financieras, conglomerados industriales que contribuyeron económicamente a la misma conferencia como Exxon Mobile, Sheel, British Petroleum, etcétera) a través de reglas claras para evaluar los compromisos que cada país asumió en su momento, como los citados INDC's de los países en vías de desarrollo y ribereños más amenazados.

La conferencia lo consiguió aprobando por unanimidad (como lo requiere la norma) el «Reglamento de París», que definió precisamente los criterios de comunicación, control y revisión de los compromisos debido a la postura contraria de naciones como Brasil, país gobernado por Jair Bolsonaro, ya que veía con preocupación las implicaciones negativas de aprobar el mecanismo de financiamiento en el área de comunicaciones y más precisamente en transportes, porque entorpecería el desarrollo económico de un área estratégica para el futuro como la soja, proceso productivo que necesita cantidades cada vez más crecientes de transportes dependientes del hidrocarburo para su envío a otras naciones como Europa o Asia Central.

De acuerdo con Mariano Morazzo, responsable de las políticas sobre el cambio climático y las energías renovables de ENEL, la COP24 de Katowice significó «un paso adelante, pequeño pero importante, porque no es fácil que todos los países se pongan de acuerdo sobre un mismo documento y, cuando eso ocurre, siempre es una buena señal» ${ }^{16}$ Sin embargo, no sucedió lo mismo en la COP25, ya que las disputas

16. «Cambio climático: ¿Qué va a ocurrir después de Katowice?», ENEL, 27 de diciembre de 2018, disponible en bit.ly/2Abt5Vi. 
sobre el mecanismo de París siguieron encontrando trabas administrativas y de negociación para encontrar el regocijo de las naciones industrializadas y sus propios conglomerados transnacionales en el resguardo de sus intereses corporativos, personales y de todo un modelo de desarrollo, anquilosado en las formas extractivistas de formación del capital financiero internacional del siglo pasado, cuando en el mundo, precisamente, lo que se necesita es una nueva forma de proyección económica y una nueva forma de producir y vivir en contacto con la naturaleza, es decir, una perspectiva ecosistémica y no antropocéntrica o egocéntrica.

En opinión de Morazzo, el papel del sector privado confirma un fenómeno sumamente importante, que tiene que ver no solo con que el compromiso de los gobiernos no es suficiente para luchar contra el cambio climático, sino también con que las vacilaciones de las conferencias sobre el clima no suponen el fracaso del actual proceso de descarbonización, aunque las expectativas formadas alrededor de la prometida, inflexible y urgente acción climática aún debe ser saldada en la $\mathrm{COP}_{26} 6$ en Glasgow. Está por verse si realmente los núcleos empresariales nacionales e internacionales están dispuestos a mayores esfuerzos por la acción climática. Es de esperar que todas estas discusiones no solo sean otro discurso cocinado y elaborado para contentar a un mundo que cada vez está en más peligro.

La gran apuesta de la pasada $\mathrm{COP}_{25}$ en Madrid era impulsar el desarrollo sustentable, sensibilizar e involucrar a la ciudadanía con la acción climática. Singularmente, de ahora en adelante, por parte de la sociedad civil, de las organizaciones no gubernamentales y de los expertos, lo crucial siempre será la acción climática de los océanos y glaciares. El objetivo no solo es fomentar el cambio de hábitos de consumo y cuidado del medio ambiente, sino también la educación en beneficio del desarrollo sostenible, que en el futuro podría modificar el modelo de desarrollo extractivista capitalista con el fin de generar nuevos modelos de consumo orientados a resultados ambientales más concretos e instaurar una gobernanza multinivel y multisectorial para la acción climática en mitigación y adaptación, lo que mejoraría nuestra institucionalidad ambiental y el desarrollo sostenible (nacional e internacional en países donde se requiera una gestión integral y multisectorial de los recursos para resguardo).

Sin embargo, muy poco de esto estuvo presente en los resultados de las negociaciones y ceremonias de clausura, ya que:

El texto finalmente adoptado por la $\mathrm{COP}_{25}$ ha descartado las referencias a la «actualización» o la «mejora’ de las Contribuciones Nacionales Determinadas (las reducciones de emisiones y las acciones de adaptación que los Estados miembros se comprometen a llevar a cabo). ${ }^{17}$

17. Margarita Mediavilla Pascal, Alberto Sanz Cobeña, Ivanka Puigdueta Bartolomé, José Luis Postigo Sierra, Mar Campins y Roberto Álvarez Fernández, «¿Una cumbre de transición? Luces y sombras de la COP25", The Conversation, 17 de diciembre de 2019, disponible en bit.ly/3gdwvq9. 
Como se ha mencionado, las esperanzas e ilusiones puestas en este trascendental encuentro por la acción climática oceánica y glaciar no fueron satisfactorias para la comunidad internacional ni para las organizaciones civiles, mucho menos para los expertos o simples ciudadanos, ya que, como fue costumbre en las anteriores versiones, la acción climática se concentró (casi) exclusivamente en el peligro que ha significado el aumento de las emisiones de gases de efecto invernadero producto del modelo de desarrollo extractivista capitalista internacional. En conclusión, todo esto llevó a que no se precisara la relación entre las emisiones y sus efectos sobre las masas oceánicas ni los cuerpos glaciares.

\section{Acción climática en torno a océanos, glaciares y la Política Oceánica Nacional}

Durante el gobierno de Sebastián Piñera, se ratificó una nueva Política Oceánica Nacional. Entre sus objetivos está fomentar la conservación del océano y el desarrollo económico sustentable repartido en diversas áreas sectoriales especiales, considerando que Chile, como país naturalmente costero, tiene al océano como futuro. En función de esto, deben realizarse mayores esfuerzos, ya que «restaurar y proteger la salud de los océanos, las costas y los recursos marinos contribuye a fortalecer la capacidad de adaptación de los sistemas naturales y humanos al cambio climático». ${ }^{18}$

La nueva Política Oceánica Nacional se firmó en febrero de 2018 bajo la presidencia de Sebastián Piñera. Al respecto, se declaró que «constituye un nuevo paso hacia la consolidación de nuestro compromiso con la gobernanza internacional de los océanos, situando a nuestro mar en el lugar predominante que le corresponde». ${ }^{19}$ En consecuencia, sus objetivos son:

- Conservación del océano y su biodiversidad.

- Desarrollo económico sustentable del océano y sus recursos.

- Soberanía y seguridad alimentaria.

- Posicionar a Chile como actor relevante en los asuntos oceánicos internacionales.

- Proteger las reservas de agua dulce.

18. Stephanie Schmidt, Bárbara Neumann, Ivonne Waweru, Carole Durussel, Sebastián Unger, Martín Visbeck, SDG14 Conserve and sustainable use the oceans, seas and marine resources for sustainable development, Comisión Económica para América Latina y el Caribe, United Nations Development Programme, Organización de las Naciones Unidas, p. 181.

19. Política Oceánica Nacional, Ministerio de Reacciones Exteriores, Ministerio de Defensa Nacional, Ministerio de Economía y Fomento, y Ministerio de Medio Ambiente, 2018, p. 7, disponible en bit. ly/2BspGlw. 
- Promover la conciencia y la comprensión sobre el océano.

- Fortalecer la institucionalidad.

- Resguardar los intereses de Chile en la Antártida.

Una Política Oceánica Nacional tiene relación con diversos temas, como conciencia marítima, desarrollo portuario, transporte marítimo, pesca, minería submarina, preservación de recursos marinos (naturales) e investigación científica marina. Esta política pretende erigirse como una en la que sitúa la «gobernanza» del océano como una política para resguardar los intereses del país ante las exigencias del cambio climático y de los objetivos de desarrollo sostenible de la Agenda 2030. La idea es poner en relieve la sustentabilidad, el aprovechamiento y el resguardo de los recursos marítimos para el futuro. Para facilitar esta política, es clave generar las bases de planificación y ejecución de nuevas políticas públicas. En ese sentido, se ha indicado que:

Se requiere equilibrar y representar intereses sectoriales y particulares ante el Estado, a través del desarrollo de un modelo institucional de gobernanza, capaz de coordinar a los distintos actores que tienen diferentes recursos y lógicas diferentes, para lograr objetivos discutidos y definidos colectivamente. ${ }^{20}$

Si bien en el papel la intención de esta nueva Política Oceánica Nacional es la de aunar los intereses multisectoriales del país que tengan relación con la biodiversidad, su conservación y con otros actores, se recalca la necesidad de diálogos con sectores clave de la actividad portuaria empresarial, lo que evidencia una falta de correlación de diálogo y participación de la sociedad civil ante el océano. Es importante mencionar que el resto de los mares continentales y océanos a nivel mundial está sufriendo los efectos nocivos de la industria extractiva, que provocan la acidificación de las aguas debido al vertido de desechos industriales que deterioran su capacidad para absorber el dióxido de carbono de la atmósfera, considerado como un factor importante por parte del V informe del Grupo Intergubernamental de Expertos sobre el Cambio Climático para las medidas de mitigación del cambio climático. Sin embargo, solo hay una escasa mención de este proceso en esta Política Oceánica Nacional, que se relaciona más con la capacidad de la actividad científica y de entes privados para mitigar sus efectos.

Para combatir el cambio, las medidas de mitigación y adaptación con respecto al océano son trascendentales para asegurar el océano a futuro, ya que para Maisa Rojas del Centro de Ciencia del Clima y la Resiliencia, el cambio climático es tan conflictivo «que no vamos a lograr enfrentarlo solo con medidas tecnológicas o con incentivos

2o. Política Oceánica Nacional, Ministerio de Reacciones Exteriores, Ministerio de Defensa Nacional, Ministerio de Economía y Fomento, y Ministerio de Medio Ambiente, 2018, p. 30, disponible en bit. ly/2BspGlw. 
económicos para los sectores productivos del país, sino que necesitamos cambios sociales profundos». ${ }^{21}$ Todo esto en relación con la necesidad de parte de la sociedad civil por instaurar de mediano a largo plazo soluciones sustentables y eficientes a futuro, en la que la gobernanza debe ser central no solo en esta política, sino en cada una de ellas donde se trate la gestión de recursos naturales, los cuales ejercen una gran importancia a escala de productividad y crecimiento económico del país que no es evidente en todas las regiones mismo, donde se privilegia tanto a la gran industria transnacional como a la nacional acuícola y pesquera, en detrimento de la pesca artesanal y de los territorios y comunidades afectadas.

Ahora, frente a la pregunta de si son capaces las instituciones ambientales de asegurar la pervivencia de las especies amenazadas en el océano por la acidificación, según las consideraciones del Informe de evaluación ambiental del 2016 de la Comisión Económica para América Latina y el Caribe, «aún persisten considerables vacíos en lo concerniente al modo de conservar las especies, ya que solo ha sido objeto de clasificaciones el $3,5 \%$ de todas las especies chilenas conocidas», ${ }^{22}$, sea por falta de voluntad de las instituciones o por la falta de apoyo presupuestario que significa la implementación y ejecución de esta política. Como acciones específicas, la Política Oceánica Nacional solo contempla desarrollar un seguimiento y una clasificación de las especies en el Océano Pacífico, e implementar «un programa de fortalecimiento institucional y creación de capacidades en materias de conservación marina». ${ }^{23}$

En la misma línea, Pilar Moraga, académica del Centro de Derecho Ambiental de la Universidad de Chile, explicó que la $\mathrm{COP}_{25}$ «es una oportunidad para reflexionar de manera profunda lo que significa el cambio climático para nuestro país y qué propuestas le vamos a hacer a la comunidad internacional para avanzar de acuerdo con las necesidades y desafíos que nos plantea este problema», ${ }^{24}$ sobre todo considerando al océano como parte fundamental del desarrollo futuro del país. Por ello, es necesario ahondar en una eficiente gestión, gobernanza y seguridad de los recursos naturales y biológicos del mar chileno para evitar su sobreexplotación a corto plazo. Para lograrlo, la Política Oceánica Nacional establece alrededor de 15 acciones específicas, entre las que destacan:

21. Francisca Palma: «Pilar Moraga y COP25. «En cambio climático se necesita más que una ley marcom", Diario UChile, 12 de abril de 2019, disponible bit.ly/3igJ3Az.

22. V Informe final de Evaluación sobre Cambio Climático y bases físicas (resumen para responsables de políticas), Resumen Técnico, Intergovernmental Panel on Climate Change, p. 82.

23. Política Oceánica Nacional, Ministerio de Reacciones Exteriores, Ministerio de Defensa Nacional, Ministerio de Economía y Fomento, y Ministerio de Medio Ambiente, 2018, p. 22, disponible en bit. ly/2BspGlw.

24. «Pilar Moraga por cambio climático: «Lo que está en juego no es el desarrollo de un país, es la humanidad»», CNN Chile, 17 de diciembre de 2018, disponible en bit.ly/2YHnHTu. 
- Desarrollar mecanismos institucionales para la investigación, monitoreo y evaluación del estado de la biodiversidad marina y costera del país.

- Integrar los intereses de las comunidades locales, comunidades indígenas y otros actores interesados en las decisiones de conservación de la biodiversidad marina.

- Implementar planes o medidas de control de la contaminación en cuerpos de agua marinos.

La promulgación de la Política Oceánica Nacional, le impone al país la urgencia de contar con mejores soluciones sostenibles en un área estratégica del desarrollo del país, aunque «el tema del océano es un tema que recién empieza a aparecer y en la práctica no se logró nada además de decir que va a estar en la agenda de las próximas reuniones», ${ }^{25}$ según afirma el científico chileno Osvaldo Ulloa, director del Instituto Milenio de Oceanografía.

El Océano Pacífico posee características que lo hacen único e indispensable, a la vez que ofrece grandes oportunidades para el desarrollo y bienestar. Además, otorga grandes facilidades y espacios para su utilización con fines ilícitos (tráfico de especies vulnerables o especies psicoactivas, pesca ilegal y seguridad de las fronteras marítimas), ${ }^{26}$ que permiten la aparición de riesgos y amenazas a la seguridad como nación. Pero es el cambio climático, como temática internacional vinculada a la biodiversidad (Ley 19.300 sobre Bases Generales del Medio Ambiente o el Convenio sobre Diversidad Biológica, promulgado mediante D.S. 1.963 de 1994 del MINREL), el que debe representar un esfuerzo sustantivo si se considera que el mar provee beneficios comerciales.

Ante los efectos nocivos del cambio climático en el océano, y como se explica en las acciones transversales que deben ser desarrolladas para potenciar la seguridad oceánica, es deber del Estado:

Elaborar planes de gestión regional y comunal como herramienta de diagnóstico, planificación y gestión participativa para el desarrollo sustentable, con énfasis en biodiversidad y adaptación al cambio climático, incluyendo las soluciones de adaptación y reducción de riesgo basadas en ecosistemas. ${ }^{27}$

25. Thomas Heselaars, «Científico nacional por acuerdo en $\mathrm{COP}_{25}$ sobre materia oceánica: «En la práctica no se logró nada»», EMOL, 17 de diciembre de 2019, disponible en bit.ly/2VtUNEp.

26. Para ello, revisar el D.S. 267 de 2005 del Ministerio de Economía, Fomento y Reconstrucción. Este documento aprueba el Plan de Acción Nacional para prevenir, desalentar y eliminar la pesca ilegal no declarada y no reglamentada en el ámbito internacional. Disponible en bit.ly/3gaCrQx.

27. Política Oceánica Nacional, Ministerio de Reacciones Exteriores, Ministerio de Defensa Nacional, Ministerio de Economía y Fomento, y Ministerio de Medio Ambiente, 2018, p. 22, disponible en bit. ly/2BspGlw. 
Todo esto con una clara visión de futuro del mar que baña al país, ya que en los ejes a trabajar se menciona como necesidad:

- Mejorar las capacidades de evaluación y adaptación del sector a fenómenos ambientales y al cambio climático en términos de la acuicultura como actividad económica preponderante del país. ${ }^{28}$

- La dimensión sustentabilidad debe estar presente en el desarrollo de acciones de innovación para enfrentar, entre otros elementos, los efectos del cambio climático y procurar que dichas acciones en los ecosistemas marinos estén de acuerdo con los principios de sustentabilidad. ${ }^{29}$

- Desarrollar y trabajar lineamientos futuros que se fomenten en la futura Política Oceánica Nacional. Estos deben observar los más altos estándares de protección al medio ambiente conforme al compromiso adquirido por Chile en el Acuerdo de París en 2016 (Fuentes, 2017: 35).

La Política Oceánica Nacional, que sucede a su homónima de 1978 con la que comparte puntos esenciales como la conservación del medio marino y la investigación científica e industria naval, actualmente sienta las bases de conservación y uso sustentable del mar y sus recursos, adaptándose a las necesidades de la globalización y a los efectos del cambio climático en el océano, así como a las áreas protegidas que Chile resguarda. De igual manera, Michelle Bachelet, expresidenta de Chile, explicó que el país multiplicó por diez la superficie marina bajo protección oficial, pasando de un $4,3 \%$ a un $42,4 \%$ en nuestra zona económica exclusiva, con una potencialidad mirando de cara hacia el Pacífico, contacto directo con los principales socios económicos de Asia.

A pesar de esto, los aportes de la sociedad civil organizada donde puedan ejercer un mayor poder decisorio en la formulación y ejecución de las políticas públicas que el país necesita frente al cambio climático en el océano aún no poseen suficiente poder para dialogar de igual forma con el resto de los actores dedicados a su formulación. Ahí, en el desarrollo de las políticas públicas, la participación de la sociedad civil solo se reduce a procesos de consulta, en los que no se toman acciones importantes ante este factor antrópico del cambio climático. Además, en el V informe del Grupo Intergubernamental de Expertos sobre el Cambio Climático, se menciona de manera genérica a la «sociedad civil», en contraste con la mención de los grupos de

28. Política Oceánica Nacional, Ministerio de Reacciones Exteriores, Ministerio de Defensa Nacional, Ministerio de Economía y Fomento, y Ministerio de Medio Ambiente, 2018, p. 28, disponible en bit. ly/2BspGlw.

29. Política Oceánica Nacional, Ministerio de Reacciones Exteriores, Ministerio de Defensa Nacional, Ministerio de Economía y Fomento, y Ministerio de Medio Ambiente, 2018, p. 40, disponible en bit. ly/2BspGlw. 
especialistas y empresas como los sectores que complementan el trabajo de la actual administración e instituciones ambientales para lograr sus objetivos.

A partir del trabajo en conjunto desde la Conferencia Oceánica del 2015 por parte del Grupo de Trabajo Interministerial que reúne a ministerios proclives a abordar la problemática ambiental, la política sobre el resguardo de los océanos, y en especial del Pacífico, ${ }^{30}$ se plantea la necesidad de desarrollar la conciencia, la participación, la información y el conocimiento sobre la diversidad marina. Sin embargo, nos hallamos en un contexto donde el instrumento principal para asegurar la participación, como el Acuerdo de Escazú, aún no es ratificado por el actual gobierno de Chile, y es esencial para monitorear los avances con respecto a los acuerdos suscritos y promesas del país para mejorar sus estándares de información, protección y preservación tanto de la ecología como de la biodiversidad marina nacional.

A pesar de todas las buenas intenciones por lograr grandes acuerdos político e institucionales (junto con la normativa chilena anterior a la Política Oceánica Nacional del 2018) en relación con la política nacional a nivel de ejes transversales, aún subsisten desafíos en la materia. Si bien es cierto que «las áreas temáticas que tocan las diferentes políticas oceánicas nacionales son diversas, en general coinciden en los temas que actualmente son prioridad, como el cuidado del ambiente marino y la investigación científica». ${ }^{31}$ Sobre este punto, la situación es distinta en la temática de los glaciares, en la que existen constantes esfuerzos de organizaciones no gubernamentales especializadas por mantener o trabajar en su seguridad, siendo este asunto solo prioridad de la Política Antártica Nacional, sin estar imbricadas en un gran corpus institucional para abordar ambos temas esenciales e imprescindibles.

El desafío a futuro es crear una sola política que involucre de forma sistemática al océano, la Antártida (o zonas oceánicas polares donde Chile tiene plena jurisdicción) y la biodiversidad conforme a las necesidades y esfuerzos internacionales para evitar mayores catástrofes ambientales. Esto se expresa, por una parte, en la nula advertencia sobre el proceso de acidificación por acción humana de esta Política Oceánica Nacional en el Pacífico, y por las metas establecidas por el objetivo de desarrollo sostenible en la Agenda 2030 con respecto a mitigar los efectos de este proceso químico influenciado por la industria y por la nula sustentabilidad en el Océano Pacífico. Esto le ha permitido a la institucionalidad ambiental chilena concentrar sus esfuerzos en:

30. «Considerando que de las 31.000 especies catastradas por el Ministerio de Medio Ambiente, entre un $22 \%$ a $25 \%$ son endémicas, y que de las 1000 especies clasificadas, más del $60 \%$ están amenazadas, se acrecienta el accountability de las instituciones fiscalizadoras de cumplir con las expectativas de asegurar la gobernanza y la seguridad de la biodiversidad marina». Para más información, véase Evaluaciones del desempeño ambiental Chile de la Organización para la Cooperación y el Desarrollo Económicos y de la Comisión Económica para América Latina y el Caribe, 2016, p. 81, disponible en bit.ly/3iewxQ9.

31. 
Integrar la adaptación al cambio climático en los sistemas de evaluación del sector público, entre otros las evaluaciones ambientales estratégicas, el Sistema Nacional de Inversión Pública y la evaluación de proyectos; integrar la resiliencia climática en el desarrollo de la regulación, las normas y los estándares pertinentes. ${ }^{32}$

Trabajar conjuntamente, y no solo a través de un plan diseñado por comisiones ministeriales segregadas sin planes en conjunto con la sociedad civil y los investigadores independientes o de organizaciones no gubernamentales, es la necesidad imperiosa si Chile pretende cumplir con los objetivos declarados, tanto de la Política Oceánica Nacional como de la Política Antártica Nacional. También es necesario que las acciones sean coherentes con el papel de Chile en las convenciones del sistema de Naciones Unidas, y en su esfuerzo ambiental internacional como país vulnerable frente a las acciones del cambio climático. De esta forma:

Resulta urgente continuar fortaleciendo la institucionalidad medioambiental antártica nacional. Se requiere de una participación efectiva en el Comité de Protección Ambiental, creado por el Protocolo para asesorar a las Reuniones Consultivas del Tratado Antártico en materia medioambiental. Asimismo, debe promoverse la entrada en vigor del Anexo VI del Protocolo relativo a la responsabilidad emanada de emergencias medioambientales, así como avanzar en la consagración de un régimen efectivo de responsabilidad por daños medioambientales en la Antártica. ${ }^{33}$

Si bien el océano es parte importante de la dinámica y del crecimiento económico del país, también debe jugar un papel fundamental en la mitigación y reducción del dióxido de carbono atmosférico, por ejemplo, a través de sus ecosistemas de carbono azul — relacionado también con los llamados «bonos azules» que se transan en el mercado como bonos de mitigación al igual que los «bonos verdes»-, que se encuentran entre los hábitats más amenazados del planeta, lo que no solo hace urgente que los países tomen una acción rápida y decidida, sino que generen políticas, planes de restauración y de conservación más intensos y ambiciosos que las actuales políticas.

Es importante considerar que los esfuerzos de la actual Política Oceánica Nacional se concentran en la sustentabilidad y la gobernanza, pero también se requiere una mayor relación con los objetivos de desarrollo sostenible del III foro y de la Agenda 2030 para potenciar este aspecto y hacer sustentable, eficiente, eficaz y transparente

32. Stephanie Schmidt, Bárbara Neumann, Ivonne Waweru, Carole Durussel, Sebastián Unger, Martín Visbeck, SDG14 Conserve and sustainable use the oceans, seas and marine resources for sustainable development, Comisión Económica para América Latina y el Caribe, United Nations Development Programme, Organización de las Naciones Unidas, p. 45.

33. Política Oceánica Nacional, Ministerio de Reacciones Exteriores, Ministerio de Defensa Nacional, Ministerio de Economía y Fomento, y Ministerio de Medio Ambiente, 2018, p. 6, disponible en bit. ly/2BspGlw. 
la gestión de los recursos del océano. La idea es que estén en simbiosis con los objetivos de desarrollo sostenible de la Agenda 2030, específicamente con el objetivo de desarrollo sostenible 14, ya que, de acuerdo con Harris (2001: 13), «la importancia de la perspectiva ecológica es cada vez más evidente, ya que la mayoría de los problemas críticos que enfrenta la humanidad surgen de fallas de la resiliencia ecológica», sobre todo en un país cada vez menos resiliente y adaptado a los efectos del cambio climático como Chile.

\section{Ley Marco del Cambio Climático}

Perfeccionar las bases de datos, con el fin de facilitar la adopción de medidas ambientales, mediante la ampliación de la recopilación y el manejo de información sobre asignación, extracción y calidad del agua, contaminación atmosférica y protección de la diversidad biológica, entre otros, y ponerla a disposición de la ciudadanía y de los organismos internacionales (OCDE, 2016: 31).34

Debido a las crisis ambientales que repercuten negativamente a futuro en la calidad de vida de pobladores en territorios de sacrificio y que son parte de la agenda Setting medioambiental en el país y en el mundo, el actual gobierno de Sebastián Piñera propone la creación de un nuevo ordenamiento jurídico medioambiental para responder ante las reclamaciones ciudadanas. El proyecto de ley por ahora está en una primera y temprana etapa de evaluación y retroalimentación por parte de la ciudadanía, a través de jornadas de reflexión o de la recepción de nuevas ideas que potencien la acción climática chilena.

Entre los objetivos declarados se destacan: i) la reducción de gases de efecto invernadero; y ii) las políticas públicas orientadas a resultados de mediano y largo plazo.

Por esto, el Ministerio del Medio Ambiente, a través de la División de Cambio Climático, está liderando el proceso de elaboración del Proyecto de Ley Marco de Cambio Climático a través de un primer proceso de consulta nacional participativo multisectorial con el objetivo de recoger la visión y la experiencia de los diferentes actores clave de la sociedad. El objetivo es recoger las necesidades y aspiraciones de la sociedad civil ante los temas de la contingencia nacional como bosques, océanos, desarrollo sostenible y la $\mathrm{COP}_{25}$.

Esta ley busca el establecimiento de principios, sistema de gobernanza, instrumentos de gestión y financiamientos adecuados — aunque las medidas de adaptación dependen de los mecanismos internacionales de préstamos, flexibilidad y transpa-

34. Para más información, véase Evaluaciones del desempeño ambiental Chile de la Organización para la Cooperación y el Desarrollo Económicos y de la Comisión Económica para América Latina y el Caribe, 2016, p. 31, disponible en bit.ly/3iewxQ9. 
rencia de los artículos 7 y 13 del Acuerdo de París- que permitan transitar tanto hacia una matriz energética carbono neutral como hacia el desarrollo sostenible, siendo este el de más difícil concreción en Chile, ya que el actual gobierno pretende instalar temas en la agenda de la próxima convención de las partes por sobre los actuales debates mundiales.

Los caminos hacia un desarrollo bajo en emisiones de gases de efecto invernadero, reducir la vulnerabilidad, aumentar la resiliencia y garantizar el cumplimiento de los compromisos internacionales son asumidos por el Estado de Chile para hacer frente a los desafíos que impone el cambio climático, pero carecen de retroalimentación efectiva por parte de la sociedad civil organizada y de la protección de sus intereses en los denominados "territorios de sacrificio», que aún deben resolverse y afectan la calidad de vida de sus habitantes. La sustentabilidad debe ser un medio para un fin superior, como lo es la convivencia entre ecología y seres humanos, ya que la sustentabilidad «significa que en nuestra elección de bienes y tecnologías debemos estar orientados a los requisitos de la integridad del ecosistema y la diversidad de especies» (Harris, 2001: 14).

Si bien desde el gobierno chileno se insiste en presentar el proyecto de Ley de Cambio Climático como la visibilización de los problemas medioambientales que afectan a las comunidades rurales y pequeñas ciudades en las que existe una gran concentración industrial, aspecto abordado a través de los planes de mitigación de gases de efecto invernadero y de la planificación de los próximos planes y proyectos del gobierno a largo plazo, como lo muestran las palabras de la ministra del Medio Ambiente, ${ }^{35}$ aún existen desafíos pendientes en la materia, pensando en el papel y las exigencias mismas de un país que presidirá la COP durante el año 2020, dirigiendo las negociaciones hacia medidas de mitigación por sobre los gases contaminantes emitidos.

Con respecto a esto, según Pilar Moraga, para construir una Ley Marco de Cambio Climático en Chile que sea responsable con las crisis actuales y que cumpla con lo pactado en el Acuerdo de París debemos:

Ir mucho más allá y eso significa establecer una meta, establecer instrumentos de cómo vamos a alcanzar ser carbono neto neutral lo más rápidamente posible, y eso requiere revisar, por ejemplo, otros proyectos de ley, como son la reforma al Sistema de Evaluación de Impacto Ambiental, como es la protección de glaciares, la reforma del código de aguas y el servicio nacional de biodiversidad. ${ }^{36}$

35. En entrevista, Schmidt señala lo siguiente: «Necesitamos avanzar hacia un país más resiliente al cambio climático. Esta ley va ser construida desde las regiones a través de un proceso altamente participativo». Para más información, véase «Qué plantea la nueva ley de cambio climático que tendrá Chile», El Mostrador, 8 de julio 2018, disponible en bit.ly/3glLXk7.

36. Francisca Palma: «Pilar Moraga y COP25. «En cambio climático se necesita más que una ley marco»», Diario UChile, 12 de abril de 2019, disponible bit.ly/3igJ3Az. 
¿Pero en qué medida esta nueva Ley Marco de Cambio Climático puede asegurar el cumplimiento de los objetivos de desarrollo sostenible relacionados a esta iniciativa? ¿Qué papel jugará la sociedad una vez que la ley pase a discusión legislativa y una vez que sea implementada? La estructura del anteproyecto depende de tres «consideraciones»:

- Que cuente con disposiciones generales que desarrollen diversos medios de implementación (estrategia de desarrollo y transferencia tecnológica; y estrategia de creación y fortalecimiento de capacidades).

- Información sobre cambio climático, acceso y participación ciudadana.

- Financiamiento para enfrentar el cambio climático (estrategia financiera, fondo de protección ambiental y presupuestos climáticos sectoriales).

Si bien la consulta ciudadana en las regiones del país apenas terminó en julio de 2019, se pretende obligar al país y a su legislación a desarrollar nuevas estrategias sobre emisiones, adaptación y resiliencia, pero esto no es suficiente tomando en cuenta las anteriores evaluaciones a las que fue sometido el país en términos de acción climática. De esta forma, se carece de una perspectiva de real gobernanza y gestión ambiental que, según la evaluación chilena medioambiental de 2016, es una de las falencias importantes a nivel de gestión de los recursos naturales en la acción por el cambio climático. El informe indica que:

- A nivel de marco regulatorio, es urgente desarrollar una política de reglamentación de las emisiones y de gestión de impuestos «verdes» y subsidios ambientales, siendo estos casos relacionados con los conflictos medioambientales en zonas de sacrificio.

- A nivel de cumplimiento y fiscalización, la urgencia de establecer un estricto régimen de responsabilidad o dolo por parte de los agentes contaminantes principalmente empresas del sector minero e industria pesada- por el daño futuro de los cuerpos de agua, el suelo, las especies y los ecosistemas; desarrollar e implementar normas y planes de rehabilitación, especialmente de suelos contaminados con metales pesados; facultar a la Superintendencia del Medio Ambiente para que haga cumplir las disposiciones sobre responsabilidad mediante medidas administrativas.

- A nivel de democracia ambiental, se argumenta la necesidad de perfeccionar el modelo de participación pública por parte de las consultas ciudadanas o de la acción de implementación y ejecución de políticas públicas con respecto a la gobernanza, uso y resguardo de los recursos naturales del país, relacionados con los territorios de sacrificio o los menos resilientes a las consecuencias del cambio climático y/o sobreexplotación de recursos naturales. 
Según el Tercer Reporte del Estado del Medio Ambiente de 2017, de las ultimas políticas ambientales con respecto al cambio climático como el nuevo Plan de Acción Nacional de Cambio Climático 2017-2022, se incluyen 96 medidas que responden, en cierto nivel, a las exigencias y recomendaciones del anterior Informe de evaluación medioambiental de la Comisión Económica para América Latina y el Caribe, pero carece de una perspectiva, línea o mesa de trabajo mancomunada sobre gestión de recursos naturales de forma integrada y de una nueva forma de gobernanza y seguridad, que posibilite al país contar con recursos naturales en el futuro.

Por su parte, en el Quinto Reporte del Estado del Medio Ambiente de 2019 (mismo año de la COP25 en Madrid), no se advierte la presencia del océano y de las masas glaciares como parte de una política integral de medidas de adaptación, mitigación o resilencia, y ni siquiera como un tema de particular interés para el Ministerio del Medio Ambiente o para los ministerios sectoriales. Esto es curioso, ya que para el Foro de Puntos Focales (instancia de discusión y debate de la última versión de la $\mathrm{COP} 25$ ) es de especial interés el tema de los océanos, las zonas costeras y sus ecosistemas, entre ellos los mega deltas, los arrecifes de coral y los manglares. Fue en este foro donde se generaron alternativas o vías de adaptación y resiliencia, se fomentó el diseño conjunto de medidas y se movilizó apoyo para la implementación de esas medidas en 2020. En relación con esto, se señaló que:

Nuestra principal prioridad es la ambición y la implementación de la acción climática. Este es un tema central, estamos en un momento histórico en el mundo, donde la población global está demandando de los gobiernos la acción para generar un cambio real en las implicancias del calentamiento global. ${ }^{37}$

Por esto, el problema que impide situar a Chile como líder de las negociaciones y de la lucha internacional contra el cambio climático es que «no cuenta con un marco jurídico que permita asignar responsabilidades de reducción de emisiones o exigir implementación y reporte de medidas de mitigación de emisiones y adaptación a los impactos del cambio climático».$^{38}$ Además, a menos que ocurra un proceso inesperado, es difícil que el gobierno de Chile pueda anteponer sus intereses ante las necesidades del resto de las naciones parte del Acuerdo de París, sobre todo cuando el tema de interés para el mundo no tenía la misma importancia para la administración de gobierno ni para la fraccionada y desagregada «política» institucional ambiental, que desarrolla sus acciones, planes y metas en marcos desapegados de la afección de la sociedad civil, las organizaciones no gubernamentales y la mirada acuciosa y experta de

37. Tomas González, «COP-25: Ministra de Medio Ambiente y ONU entregan detalles de la denominada «Blue COP»», Diario UChile, 26 de junio de 2019, disponible en bit.ly/3igDb8v.

38. Informe Anteproyecto Ley Marco del Cambio Climático, Ministerio de Medio Ambiente, 2019, p. 2, disponible en bit.ly/2NIyoQA. 
académicos nacionales e internacionales, desafectados todos ellos de participar de un proceso común de resguardo al medio ambiente y de lo que, en última consideración, le ha dado grandes réditos comerciales a la «industria» nacional.

Considerando esto, Chile no cuenta con un marco jurídico que permita asignar responsabilidades de reducción de emisiones o exigir implementación y reporte de medidas de mitigación de emisiones y adaptación a los impactos del cambio climático (ni contando su impacto en el océano de forma concreta y coherente con una política ambiental). Las razones por las cuales es necesaria una Ley Marco de Cambio Climático son:

- La necesidad de la gobernanza climática, que otorgue facultades y obligaciones de los organismos del Estado para la acción climática tanto a nivel vertical (nacional a municipal) como a nivel horizontal (distintos sectores).

- El deber de institucionalizar la visión del Estado, estableciendo en la ley la meta de carbono neutralidad.

- Es necesario permitir la flexibilidad y autonomía necesaria para establecer las acciones específicas según las necesidades, los territorios afectados y dependientes de las condiciones cambiantes en el tiempo.

Esto es necesario en un país sumamente vulnerable a la afección del cambio climático en la biodiversidad terrestre, oceánica y antártica. Además, puede contribuir decisiva y positivamente si se hace desde una perspectiva multisectorial con todos los actores en juego en base a las medidas de adaptación, resilencia, mitigación y sostenibilidad que el país requiere para afrontar sus propias apuestas en torno a la carbono neutralidad (de acuerdo con las proposiciones del Acuerdo de París desde su origen en la $\mathrm{COP}_{21}$ ). Todo esto aunque algunos sectores empresariales neoliberales renieguen de sus evidentes impactos positivos y beneficios, ya que «el proyecto en sí establece metas ambiciosas que podrían tener efectos negativos en la economía, sin implicar una contribución negativa que haga frente al calentamiento global», ${ }^{39}$ porque de ser así, la Ley Marco de Cambio Climático afectaría el sistema extractivo que ha dado cuantiosos dividendos comerciales a los círculos «industriales» (cuando no hay industrias en Chile con alto valor agregado y que sean parte de las cadenas internacionales de valor corporativistas del mundo capitalista) detentados por unos pocos y sostenidos en un modelo neoliberal rentista de pocos incentivos, inversiones e investigación, que hace gala de su «eficiencia y productividad» y que solo reporta beneficios a unos pocos burgueses.

A pesar de esta demostración negativa por parte del empresariado corporativista

39. «Ambiciosa Ley Marco de Cambio Climático en un contexto de recesión mundial», Diario Constitucional, 19 de mayo de 2020, disponible en bit.ly/2BTDgy8. 
capitalista de realizar cambios significativos en favor de la fuentes de sus onerosos ingresos (el ecosistema en sí), el problema de los océanos y su vinculación con la crisis climática mundial fue un tema transversal desde la COP21 en París hasta la actualidad (aunque con significativas diferencias de intereses en la pasada COP25), ya que, como se mencionó en la introducción de este artículo, la población mundial depende de los océanos de más maneras de las que se piensa.

\section{Conclusión}

La acción climática nacional y mundial demanda voluntades de todos. Estas voluntades no solo deben ser políticas, sino también de las instituciones, de diputados y senadores, de secretarios, subsecretarios y ministros. A pesar de que durante el Protocolo de Kioto y el de Copenhague la ciencia fue duramente cuestionada en relación con la real dimensión del cambio climático, ya no hay marcha atrás y el tiempo se agota. Según el V Informe del Grupo Intergubernamental de Expertos sobre el Cambio Climático, se detalla que «la temperatura media global muestra un incremento de $0,85^{\circ} \mathrm{C}$ (entre $0,65^{\circ} \mathrm{C}$ y $1,06{ }^{\circ} \mathrm{C}$ ) en el período 1880-2012» (Rodríguez, Picatoste y Heras, 2014: 36), y que su tendencia es seguir aumentando. Los océanos y las masas glaciares deben tener su lugar en las discusiones futuras de los Puntos Focales y en la COP26 de Glasgow, así como en otras instancias que permitan que países atrasados en esa materia, como Chile, puedan trabajar conjuntamente en buscar soluciones eficientes frente a los impactos (al parecer) inadvertidos del cambio climático al océano y el territorio antártico.

El proceso de consulta pública de la Ley Marco de Cambio Climático se enmarcó entre el 18 de junio y el 31 de julio de 2019. La ciudadanía hizo 1.475 observaciones al anteproyecto, por lo que han existido avances en la matera a nivel nacional. Sin embargo, de acuerdo con el artículo 4 del Acuerdo de París, se requieren mayores ambiciones para lograr la carbono neutralidad, lo que ya ha sido declarado como política pública en países como Suecia y Noruega. Sin embargo, en nuestro país aún no hay una base suficiente para abordar de manera urgente la integridad de los ecosistemas y territorios afectados, ya que en el anteproyecto de la ley se estableció el límite de implementación hacia el 2050, lo que no satisface la necesidad de los «territorios en sacrificio» por alcanzar metas más próximas y un trabajo más eficiente, eficaz y transparente desde el ejecutivo.

A pesar de que en el acuerdo de la $\mathrm{COP}_{21}$ el país se comprometió con mayores ambiciones a nivel nacional con respecto a la transición hacia una matriz energética carbono neutral y que apueste por energías renovables y limpias, esta investigación sostiene que el país carece de la voluntad política para hacer partícipe a la ciudadanía de la política ambiental y de iniciativas suficientes que aprovechen las ventajas comparativas de Chile para potenciar las energías limpias, exigencias que fueron plantea- 
das en el Acuerdo de París y que no son suficientes para llevar al país hacia el objetivo del desarrollo sostenible antes de cumplir con los compromisos de la Agenda 2030.

A pesar de que la $\mathrm{COP}_{25}$ fue catalogada como una «decepción», lo único destacable fue poner a las masas oceánicas como otro punto a considerar en la acción climática mundial. El asesor senior de Ciencia Marina y Conservación del Programa Polar Marino y Global de la Unión Internacional para la Conservación de la Naturaleza, Dan Laffoley, reclama a los gobiernos que deben actuar con la mayor ambición para recortar emisiones y para reducir todos los factores que estresan hasta el límite al océano. El objetivo de esto sería evitar quedarse sin «una de las mayores armas» para luchar contra este desequilibrio «catastrófico» para la civilización.

El problema fundamental de Chile, que impide situarlo en la $\mathrm{COP}_{25} 5$ y en la futura $\mathrm{COP}_{2} 6$ de Glasgow como un líder en las negociaciones y en la lucha internacional contra el cambio climático, es que «no cuenta con un marco jurídico que permita asignar responsabilidades de reducción de emisiones o exigir implementación y reporte de medidas de mitigación de emisiones y adaptación a los impactos del cambio climático».40 A menos que ocurra algo inesperado, es difícil que el gobierno de Chile pueda anteponer sus intereses ante las necesidades del resto de las naciones parte del Acuerdo de París, sobre todo cuando:

No podemos seguir en la lógica de avanzar en la medida de lo posible, cuando la vida de muchas especies biológicas (incluidas muchas comunidades humanas) está en juego. Nuestro país no es la excepción, somos extremadamente sensibles a los efectos del cambio climático y, por tanto, candidatos a sufrir sus efectos o sea tener daños severos. ${ }^{41}$

\section{Referencias}

Di Pietro (2019). «Acuerdo de París: ¿Nuevos compromisos con el medio ambiente o nuevas oportunidades de negocios?» Revista Estudios Internacionales, 51 (192): 57-70. DOI: 10.5354/0719-3769.2019.52814.

Fuentes, Guido (2017). «Una política oceánica para Chile». Revista de Marina, 956: 32-35. Disponible en bit.ly/3e $\mathrm{U}_{5} \mathrm{KH}_{3}$.

HARRIS, Jonathan (2001). Basic principles of Sustainable Development. Washington D.C.: Island Press.

Rodríguez, Ernesto, José Ramón Picatoste Ruggeroni y Francisco Heras Hernández

40. Informe Anteproyecto Ley Marco del Cambio Climático, Ministerio de Medio Ambiente, 2019, p. 2, disponible en bit.ly/2NIyoQA.

41. Flavia Liberona, «Reunión climática acentúa el desafío de Chile de ser líder internacional en cambio climático con la COP25», Cambio Climático Chile, disponible en bit.ly/3g4AGUQ. 
(2014). «Quinto informe de evaluación del IPCC: bases físicas». Tiempo y Clima, 5 (4): 36-40. Disponible en bit.ly/3g8icmI.

\section{Sobre el autor}

Pablo Carrasco Hernández es licenciado en Historia por la Universidad Diego Portales y magíster en Estudios Internacionales por la Universidad de Chile. Su correo electrónico es pablocarrascoh15@gmail.com. 
La Revista de Derecho Ambiental, del Centro de Derecho Ambiental de la Facultad de Derecho de la Universidad de Chile, es un espacio de exposición y análisis en el plano académico del derecho ambiental. Su contenido se presenta a través de doctrina, jurisprudencia y recensiones, y aborda diversas materias relacionadas con la gestión, institucionalidad y herramientas de protección ambiental y desarrollo sustentable. Se presentan artículos de diferentes autores, en los que se analizan y abordan casos y temas jurídico-ambientales de creciente interés y actualidad.

\author{
DIRECTORA \\ Valentina Durán Medina \\ EDITORES \\ Jorge Ossandón Rosales \\ y Antonio Pulgar Martínez \\ SITIO WEB \\ revistaderechoambiental.uchile.cl \\ CORREO ELECTRÓNICO \\ revistada@derecho.uchile.cl \\ LICENCIA DE ESTE ARTÍ́CULO
}

Creative Commons Atribución Compartir Igual 4.o Internacional

La edición de textos, el diseño editorial

y la conversión a formatos electrónicos de este artículo

estuvieron a cargo de Tipográfica

(www.tipografica.io). 